\title{
Acute confusion in a cyclist
}

\author{
Dr Angeliki Giannopoulou, Dr Akhila Mallipedhi, Dr Sam Rice \\ Department of Endocrinology, Hywel Dda University Health Board
}

\section{Case presentation}

A 46-year-old male was brought to the hospital by his uncle as he was found to be acutely confused and agitated 'talking rubbish' and giggling inappropriately. Last time he was seen well was three days ago. He was a cyclist who lived alone and his past medical history included Graves' disease and atrial fibrillation. He was on propranolol, carbimazole and warfarin. A month ago he needed admission to a different hospital after falling off his bike. At that time he also developed confusion and had undergone computed tomography (CT) and magnetic resonance imaging (MRI) of his brain which were normal. A lumbar puncture (LP) and an electroencephalogram (EEG) had excluded herpes encephalitis and the symptoms eventually had been attributed to opioid analgesics. On this admission, he was apyrexial, tachycardic, overtly confused and incoherent. He had prominent exopthalmos and fine tremor in both arms with brisk reflexes. The rest of the examination was unremarkable.

\section{Investigations}

He had a chest X-ray and a CT head which were normal. From laboratory findings, there was mild leukocytosis and the rest of septic screen was normal. His thyroid stimulating hormone (TSH) was $0.01 \mathrm{mU} / \mathrm{L}(0.27-4.2 \mathrm{mU} / \mathrm{L})$ and free thyroxine (fT4) was $43.2 \mathrm{pmol} / \mathrm{L}(11.0-25.0 \mathrm{pmol} / \mathrm{L}$ ). In order to complete the investigations for acute confusion and rule out an organic cause an LP and an MRI were attempted but the patient was too confused and agitated to tolerate any further tests.

His confusion was felt to be secondary to the thyrotoxicosis and he was referred to the Endocrine team. Psychosis secondary to mental health illness was also queried. The Endocrinologists advised to increase the carbimazole to $40 \mathrm{mg}$ once a day (OD) and check his anti-thyroid peroxidase antibodies (anti-TPO) which came back remarkably raised at $>600 \mathrm{U} / \mathrm{ml}(<34.0 \mathrm{U} / \mathrm{ml})$.

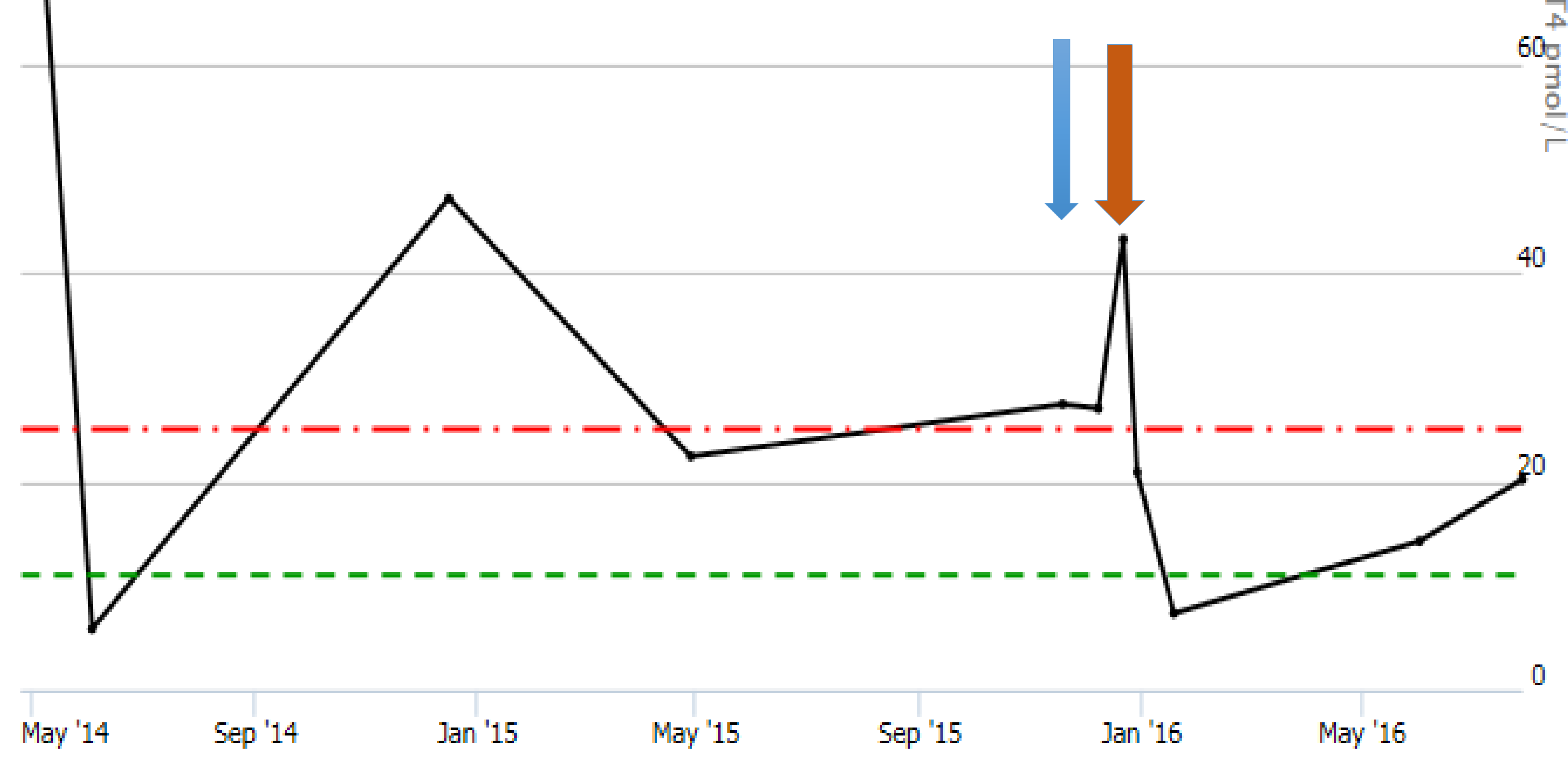

Red arrow : Free T4 on this admission

Blue arrow: Free T4 on previous admission with same symptoms
Diagnosis and Management plan

The diagnosis of Hashimoto's encephalopathy was made and the patient improved dramatically after having $20 \mathrm{mg}$ of prednisolone OD. His thyroid function tests (TFTs)s a few weeks after were within range and as he was not a candidate for radioactive iodine, in view of his active ophthalmopathy, he had a partial thyroidectomy. He made a good recovery and currently is on thyroxine replacement and weaning dose of steroids.

\section{Discussion}

'Hashimoto's encephalopathy' (HE) is the term used to describe a wide range of neurological presentations associated with autoimmune thyroid disease such as Hashimoto thyroiditis, Grave's disease and idiopathic thyroid failure. The presence of elevated titres of anti-thyroid antibodies, ,predominantly anti TPO but also antithyroglobulin antibody (anti-TG Ab), and a dramatic respone to immunosuppression can be conclusive. It is a rather rare condition, usually underdiagnosed. The mechanism which leads to HE remains unknown but there is indirect evidence which supports an autoimmunopathology e.g female prevalence, association with autoimmune disorders, relapsing-remitting clinical course and good response to immunosuppression(1). Reports from some autopsies have showed perivascular lymphocytic infiltration demonstrating a vasculitic aetiology (2) while other hypotheses suggest that cerebrospinal fluid thyroid autoantibodies react with intracranial antigens forming localised immune complexes $(3,4,5,6)$. Looking back to our patient's previous admission, again with confusion, it was felt now that his symptoms even then were consistent with $\mathrm{HE}$. His free T4 was only slightly raised (see graph) but $\mathrm{HE}$ is independent of the thyroid status. Therefore, normalisation of the thyroid hormone levels alone will not cure it and relapsing neurologic symptoms can be expected without immunosuppressive therapy (3).

\section{Conclusion}

in patients presenting with altered behaviour and when other aetiologies have been excluded, raised anti thyroid antibodies can provide the diagnosis of Hashimoto's encephalopathy. Prompt diagnosis is important as it is a life threatening condition which is however highly responsive to steroids with an impressive resolution of the symptoms.

\section{References}

1.Carlone C, Todini L, Marini I, Majorana M, Casciato S, Giallonardo AT, Pallagrosi M, Salviati M, Biondi M. Acute psychiatric presentation of steroid-responsive encephalopathy: the underrecognized side of autoimmune thyroiditis. Riv Psichiatr 2013;48 (2):169-173

2. Nolte KW, Unbehaun A, Sieker H, Tm Kloss, Paulus W. Hashimoto encephalopathy: a brainstem vasculitis? Neurology 2000 54:769-770.

3. Huang W, Xia C, Chatham M. Infectious disease or Hashimoto's Encephalopathy flares: A case report. Seizure 20 (2011) 717 719 .

4. Chaudhuri A, Behan P.O. Hashimoto's encephalopathy: a relapsing form of acute disseminated encephalomyelitis. J Neurol SCi 2005; 235: 75-76.

5. Ferracci F, Moretto G, Candeago RM, Cimini N, Conte F, Gentile M et al. Antithyroid antibodies in the CSF: their role in the pathogenesis of Hashimoto's encephalopathy. Neurology. 2003; 60: 712-714.

6. Gini B, Lovato L, Cianti R, Cecotti L, Marconi S, Anghileri E et al. Novel autoantigens recognized by CSF IgG from Hashimoto's encephalitis revealed by a proteomic approach. J Neuroimmunol. 2008; 196: 153-158. 